\title{
Situation of Rabies in the Republic of Chad
}

\author{
Bidjeh Kebkiba ${ }^{1,}$, , Mahamat Ouagal ${ }^{1}$, Mahamat Makoundji Kourdina ${ }^{1}$, Naïssengar Kemdongarti ${ }^{1}$, \\ Rollande Mindekem², Oussiguéré Assandi ${ }^{1}$ \\ ${ }^{1}$ Department of Animal Health, Virology, Bacteriology and Epidemiology Units, Livestock Research Institute for Development (IRED), \\ N'Djamena, Chad \\ ${ }^{2}$ Support Center for International Health (CSSI), N'Djamena, Chad
}

Email address:

bidjehkebkiba@yahoo.fr (B. Kebkiba),bidjeh.kebkiba@gmail.com (B. Kebkiba)

${ }^{*}$ Corresponding author

\section{To cite this article:}

Bidjeh Kebkiba, Mahamat Ouagal, Mahamat Makoundji Kourdina, Naïssengar Kemdongarti, Rollande Mindekem, Oussiguéré Assandi. Situation of Rabies in the Republic of Chad. Animal and Veterinary Sciences. Vol. 5, No. 4, 2017, pp. 52-56. doi: 10.11648/j.avs.20170504.11

Received: June 1, 2017; Accepted: June 12, 2017; Published: July 17, 2017

\begin{abstract}
Known since the highest antiquity, rabies is a viral, mortal disease. Rabies affects all mammals, including humans, and is a threat to both public and animal health. Rabies is therefore a zoonosis. Rabies is caused by a virus belonging to the family Rhabdoviridae and gender lyssavirus. Rabies virus is an enveloped virus presenting in an electron microscopy as a shell. It is a viral disease that is characterized by encephalitis. It is poorly controlled and even increasing in many countries in Africa and Asia. In Chad, rabies is enzootic, and its diagnosis is only possible at the Veterinary and Zootechnical Research Laboratory of Farcha (LRVZ), the current Livestock Research Institute for Development (IRED). In intertropical Africa, rabies is urban; it is carried by dogs that represent the reservoir of the wild virus and the vector of the disease in humans because of the close ties they keep with humans. Other domestic animals do not intervene much. This manuscript describes the evolution of the epidemiological situation of rabies in the Republic of Chad and the results of the studies carried out in this field from 1990 to date. The results of the mass and free vaccination campaigns undertaken in 2003, 2012 and 2013 gave a vaccination coverage rate of $78,70.83$ and $71 \%$ respectively; On the other hand, the mass and paid vaccination campaign gave a vaccination coverage rate of $23 \%$. Thanks to the results of the two mass vaccination campaigns for dogs and other companion animals, the incidence of rabies in N'Djamena drops from four (4) new cases per month in 2012 to less than two cases per month in 2014. Although efforts were made to control the diease, the existing data on canine rabies in Chad are fragmentary and do not allow accurate description of the disease situation throughout the country. To effectively control rabies in Chad, mass vaccination campaign for dogs and other companion animals must be free of charge throughout the all national territory, since the fight against rabies is a public good, an ethical imperative that can contribute to the fight against poverty. Emphasis should therefore be put on raising public awareness by using all possible means, such as radio, television, newspapers and awareness-raising meetings.
\end{abstract}

Keywords: Canine Rabies, Virus, Diagnosis, Vaccination, Immunofluorescence, Immunohistochemical Test, N'Djamena, Chad

\section{Introduction}

Few are diseases, which cause as much fear as rabies. This applies not only to people who are bitten, but also to public health and animal health entities responsible for preventing and controlling the disease. World Health Organization [1, 30] noted that more than 95 per cent of cases of human rabies caused by dog bite. Rabies is usually caused by the bite of an infected animal. Rabies, when declared, is always fatal, because there are still no effective drugs to treat it, but preventive treatment based on immunization exists [22]. There is therefore a preventive medication immunizing practiced without any bite.

Rabies is a zoonosis that is as much a threat to public health as other infections. According to World Health Organization [2], more than 3 billion people in more than 
150 countries and / or territories are at risk of contracting rabies. The disease kills over 60,000 people a year worldwide [20]. According to data reported to the World Health Organization, in 2004 the estimated number of deaths caused by rabies exceeded 55,000 worldwide, with more than 90 per cent occurring in Africa and Asia [3]; Africa and Asia are the areas most affected by the disease. In addition, more than 30 to 40 per cent of rabies deaths occur in children under 15 years of age $[3,4]$. According to WHO [1], rabies is the tenth cause of human death in the world among infectious diseases.

In Africa in general and in Chad in particular, dogs are the main vectors, but cats and other pets can also contract the disease and transmit it to humans. In Chad, the risk of human rabies is higher because more than 70 per cent of the population lives in poverty, and the cost of post-exposure prophylaxis is also very higher than the minimum wage [17]. There is also the non availability of the vaccine in country; most often, you have to go to neighboring countries to get them. According to the reports of the Urban Veterinary Clinic of N'Djamena from 1990 to 1994 [29], cases of dog bites are frequent in N'Djamena, but are not all reported to local Veterinary Authorities. Every year, many victims of dog bites are registered in the city of N'Djamena. In this city, rabies studies began in 2000 and proved that many people are victims of dog bites $(25,17)$. The purpose of this manuscript is to describe a canine rabies situation in The Republic of Tchad since 1990 to date.

\section{Materials and Methods}

The information summarized in this manuscript is extracted for most of the annual reports of the Veterinary and Zootechnical Research Laboratory of Farcha (LRVZ), the current Livestock Research Institute for Development (IRED), the urban veterinary clinic of N' Djamena as well as the reports of the mass and free vaccination campaigns of dogs and other pets carried out in 2003 then in 2012 and 2013 in the city of N'Djamena.

\subsection{Historical}

Rabies study began in Chad since the colonial era. In 1963, a case of rabies was observed in the city of Abougoudam located in the east part of Chad. This case was confirmed by LRVZ.

From 1969 to1970, Sirol and Provost observed 6 cases of human death due to rabies at the central hospital of Fort Lamy. [28]

Studies conducted by Veterinary and Zootechnical Research Laboratory of Farcha on pathological dominants classified rabies as one of the major infectious diseases in the country [12]. The study on rabies conducted in N'Djamena from 2000 to 2002 by the same Institution, Swiss Tropical and Public Health Institute (Swiss-TPH) and Support Center for International Health (CSSI) showed that rabies is rampant in this city and other parts of Chad in an enzootic way. Thanks to the scientific work carried out by these three institutions, it is now known that the incidence of canine rabies in N'Djamena is $1.4 \%$ [5]. Based of the results of these studies and the pilot mass vaccination campaign carried out in 2003, the results of pilot free and then paid vaccination campaigns, and results of study of canine demography impact on rabies transmission [14, 16, 19], the Directors of LRVZ, Swiss-TPH and CSSI signed a Memorandum of Understanding on 28th September 2012 in which stipulated mass and free anti-rabies vaccination campaign in the city of N'Djamena. The objectives of the mass and free vaccination campaign for dogs and other pets were to: break the cycle of rabies transmission among the canine population in N'Djamena and vaccinate at least 70 per cent of the canine population of N'Djamena for two (2) consecutive years (2012-2013).

Organization of mass and free vaccination campaigns of 2012 and 2013 was carried out by a coordination committee made up of representatives of the three partners (LRVZ, Swiss-TPH, and CSSI) and supervisors. Among the competences and responsibilities of this committee were the decision on the spatial progress of the vaccination campaign, organization of awareness, logistics and communication with the District authorities. This committee meets twice a week, on Tuesday and Thursday, and in case of major difficulties, it reports to the monitoring committee. The Monitoring Committee consists of the directors of the three institutions: LRVZ, Swiss-TPH and CSSI or their respective representatives. The campaign of mass and free vaccination of dogs and other pets concerned first two Districts, then all the 10 Districts that counts the city of N'Djamena. To carry out mass and free vaccination campaigns in 2012 and 2013, 50000 doses of rabies vaccine (rabisin), $50 \quad 0002 \mathrm{ml}$ syringes, 50000 needles and 50000 collars for marking vaccinated animals and 10 registers have been deployed. Ten (10) 5-liter coolers for transporting and storing the vaccine and three (3) 50-liter coolers were also purchased. It should be noted that the 50,000 vaccination books were provided free of charges by Merial. Vaccines stamps with the campaigns $\operatorname{logo}$ to authenticate vaccination books were purchased. Three four wheels drive vehicles (two from LRVZ and one from CSSI) were made available to facilitate the transport of vaccinators, refueling vaccination teams in the event of a vaccine breakdown or consumables, and distributing the snack at noon. Each supervisor was equipped with a vehicle. Also, the public awareness campaign on the danger of rabies in the population of N'Djamena was organized in two stages. The first stage preceded the official launch of the campaign. It consisted of placing posters in public places (hospitals, health centers, schools and borough offices, etc.), the distribution of leaflets in hospitals and health centers; the second stage consisted to hold meetings with district authorities.

\subsection{Diagnostic}

In general, diagnosis of rabies consists in recognizing the existence of acute encephalomyelitis in the animal and linking its cause to its specific agent, which is rabies virus. In fact, in the fight against this scourge, laboratory plays a fundamental 
role because only the results of the diagnosis can objectively guide if you have to undertake or not post-exposure treatment and to introduce complex measures to stop the outbreak. Despite the importance that has this component in the fight against rabies, to date, diagnosis of rabies is done at IRED, which is the only diagnostic laboratory of rabies in Chad.

In the past (prior to 1998), rabies diagnostic in Chad was based on Negri bodies identification in histological sections using Sellers and Mann staining methods of Pascu Atanasiu et al. [11]. From 1999 to 2000, the detection of Negri bodies in histological sections was followed by inoculation to 3-5 weeks old mice (in cases of negative results). At the same period and with the advancement of new technologies and the frame of strengthening of diagnostic capacities, LRVZ sent one technician to Nancy (France) for training on rabies diagnostic. It was then on the return of this technician that the Rabies Diagnostic Unit opened its doors. Since then, diagnostic of canine rabies is made by direct immunofluorescence test (IFT) described by Bourhyet al. [8].

In 2012, LRVZ, in collaboration with CDC (Center of Diseases Control) of USA, wanted to introduce direct and rapid immunohistochemical test (DRIT) into the rabies diagnostic system in Chad. This test is based on a peroxidase histochemical reaction. To this end, a training workshop was organized in N'Djamena for field workers. The objective of the workshop was to decentralize diagnostic of canine rabies in Chad. Unfortunately, the World Organization of Animal Health (OIE) failed to validate the kit and also due to lack of cold chain in the field for kit storage, the kit was not available on the field. Since 2013 canine rabies Diagnostic Unit at IRED is validating the rapid rabies diagnostic test called «ANIGEN», which was also validated by Institut Pasteur in Paris in 2015. The said test is easy to perform and does not need a cold chain in the field. Its utility in the field was proved [26]. Apparently, the test works well because all the samples tested have all been positive for the direct immunofluorescence test.

\section{Results}

Results of rabies diagnosis in LRVZ from 2000 to 2012 show that out of 536 samples received, $303(85.11 \%)$ were positive, $50(14.04 \%)$ were negative and 03(0.84\%) were doubtful. Table 1 shows the rabies situation in the city of N'Djamena from 1992 to 1994 . From this table, it can be seen that in three years, 289 cases of rabies were presented to urban veterinary clinic of N'Djamena, 231(79.93\%) of which were positive, 1259 persons have been bitten by dogs and 253 persons were under treatment.

Table 1. Situation of canine rabies in N'Djamena (1992-1994).

\begin{tabular}{lllll}
\hline Years & \multicolumn{2}{l}{$\begin{array}{l}\text { Preseented cases to } \\
\text { urban veterinary clinic }\end{array}$} & $\begin{array}{l}\text { Persons under } \\
\text { treatment }\end{array}$ & $\begin{array}{l}\text { Bitten } \\
\text { persons }\end{array}$ \\
\hline 1992 & 258 & $(206+)$ & 82 & 464 \\
1993 & 25 & $(20+)$ & 137 & 596 \\
1994 & 6 & $(5+)$ & 34 & 199 \\
Total in 3 years & 289 & $(231+)$ & 253 & 1259 \\
\hline
\end{tabular}

Sources: Annual reports of urban veterinary clinic of N'Djamena
Table 2 shows the situation of rabies in the city of N'Djamena from 2004 to 2008. From this table, it can be noted that in five years, 561 dogs were vaccinated by the urban veterinary clinic of N'Djamena. During the same period, clinic recorded 539 biting dogs among which 22 were declared rabid and 34 suspected rabies. Out of $\mathrm{f}$ the 539 registered biting dogs, 42 are stray dogs. Out of the 571 persons, who were bitten, only $138(24,16 \%)$ were treated. It should be noted that in five years, only one person died of rabies.

Table 2. Canine rabies situation in N'Djamena (2004-2008).

\begin{tabular}{lllllll}
\hline & $\mathbf{2 0 0 4}$ & $\mathbf{2 0 0 5}$ & $\mathbf{2 0 0 6}$ & $\mathbf{2 0 0 7}$ & $\mathbf{2 0 0 8}$ & Total \\
\hline Biting animals & 143 & 128 & 91 & 69 & 98 & 539 \\
Bitten persons & 153 & 140 & 96 & 72 & 100 & 571 \\
Bitten by stray dogs & 16 & 12 & 6 & 4 & 4 & 42 \\
Persons under treatment & 54 & 57 & 4 & 26 & 17 & 138 \\
Deaths from rabies & 0 & 0 & 1 & 0 & 0 & 1 \\
Suspected rabid dogs & 2 & 11 & 3 & 8 & 10 & 34 \\
Dogs declared rabid & 8 & 7 & 1 & 4 & 2 & 22 \\
Antirabies vaccination & 149 & 81 & 78 & 139 & 114 & 561 \\
\hline
\end{tabular}

Sources: Annual reports of urban veterinary clinic of N'Djamena

In 2003, 3000 doses of vaccine offered by Merial allowed to vaccinate in 3 zones of dogs concentration in N'Djamena for 2 days each. This restricted campaign of free vaccination gave a vaccination coverage rate of 78 per cent [6], compared with the pilot vaccination campaign at a cost of $2000 \mathrm{~F} / \mathrm{CFA}$ per animal whose vaccination coverage was $23 \%$ [7]. the pilot free dog vaccination campaign against rabies in 2003 in N'Djamena demonstrated that the canine population can be vaccinated and that vaccination coverage can reach 70 per cent (threshold prescribed by the OIE) to break the epidemiological cycle of the virus [13]. Thanks to the scientific work carried out by LRVZ, Swiss-TPH and CSSI, the incidence of canine rabies in the city of N'Djamena is known to be $1.4 \%$ o per year [5]. Of the 10 districts covered by the 2012 antirabies campaign, a total of $18,182(70.83 \%)$ dogs were vaccinated. Apart from the dogs, 1,484 cats and 104 monkeys were also vaccinated [9].

In accordance with the terms of a protocol signed in 2012 between the three institutions involved in the control or even eradication of rabies in Chad, a second free mass vaccination campaign was organized in 2013 by Livestock Research Institute for Development in partnership with Swiss-TPH and CSSI. Vaccination coverage in the 2013 was estimated at $71 \%$.

Following these two mass and free vaccination campaigns for canine and other pets canine rabies incidence dropped by $90 \%$ in less than one year [27]. Therefore, at the end of the intervention in 2014 rabies cases were only reported from peripherical zones of N'Djamena. Recognizing the resurgence of canine rabies cases in the 9th District of N'Djamena City and Koundoul, a locality located at $20 \mathrm{~km}$ from Ndjamena, a final anti-rabies campaign was organized in these two areas to prevent the reintroduction of the canine rabies virus in the other districts and thus secure the entire city. This campaign took place in 2016. During this campaign, 5,619 dogs, 594 cats and 15 monkeys were vaccinated. 


\section{Discussion}

The mass and free vaccination campaigns for dogs and other pets organized in 2012 and 2013 reached a vaccination coverage rate of 70.83 per cent and 71 per cent, respectively, and the pay campaign 23 per cent. This demonstrates the problem of low purchasing power of the population against rabies and the possible failures of a vaccination campaign with such approach. In any case, all these efforts made in recent years show the will of Chad to control or even eradicate rabies from its territory. Vaccination of dogs and other pets, the only effective option for controlling canine rabies, deserves special attention without losing sight of the availability and accessibility of post-exposure human vaccine in cases that occur.

The low number (561) of dogs vaccinated in five years by the urban veterinary clinic is explained by the fact that the vaccines are paid (3500f / CFA per dose) and population cannot efford that.

The resurgence of canine rabies cases recorded in the 9th District is explained by the fact that this District borders with the locality of Koundoul and the town of Kousseri (located at other side of the river chari), which had not been affected by the two campaigns of mass and free vaccination of dogs And other pets. Rabies virus would have entered into the 9th District from these two cities by stray dogs or by not confined owned dogs.

Results of studies conducted elsewhere and in Chad [10, 21, 24 and 27] have shown that if approximately 70 per cent of the canine population is vaccinated against rabies, the transmission cycle of the rabies virus may be broken. In this sense, the constraints linked to the effective control of rabies are not of a technical nature but rather of an economic and logistic [15]. It should be noted that rabies declared clinically is inevitably fatal.

\section{Conclusion}

Although efforts were made to control the diease, the existing data on canine rabies in Chad are fragmentary and do not allow accurate description of the disease situation throughout the country. The results of mass and free vaccination campaigns of dogs and other pets organized in 2003, 2012 and 2013 were satisfactory because threshol $(70 \%)$ prescribed by OIE were reached.

To effectively control rabies in Chad, mass vaccination campaign for dogs and other companion animals must be free of charge throughout the all national territory, since the fight against rabies is a public good, an ethical imperative that can contribute to the fight against poverty. Emphasis should therefore be put on raising public awareness by using all possible means, such as radio, television, newspapers and awareness-raising meetings.

\section{Acknowledgments}

The authors express their profound gratitude to the UBS Optimus Funds of the Swiss Federal Veterinary Foundation and to the General Directorate of IRED for their contribution to the financing of the activities of the two campaigns of mass and free vaccination of dogs and other pets in the city of N'Djamena (2012 and 2013). They sincerely thank the Ministry of Livestock and Animal Production for authorizing the implementation of this work.

Our thanks also go to the General Directorate of Veterinary Services, the National School of Livestock Techniques (ENATE) and the Regional Delegation for Livestock in N'djamena, as well as the Urban Veterinary clinic in N'Djamena for their frank collaboration.

Authors thank District authorities, Owners of dogs for their collaboration and active participation in the mass and free vaccination campaigns held in 2003, 2012, 2013 and 2016.

Our deepest gratitude goes also to vaccinators, supervisors and drivers for their active participation in vaccination campaigns.

The authors are grateful to all those, who have contributed to the good progress of canine rabies elimination activities in Chad and vaccination campaigns.

\section{References}

[1] World Health Organization- WHO, (2010). Weekly epidemiological record, 85, 32, 309-320.

[2] World Health Organization- WHO, (2013). Expert consultation on rabies, second eport.

[3] World Health Organization- WHO, (2004). Rabies [archive] available from www.who.int/fr.

[4] Rabiesblueprint.com. [Internet]. 2011 Sep 24 [cited 2011 Sep 24]; Available from: http://www.rabiesblueprint.com/.

[5] Kayali U, Mindekem R, Yemadji N, Oussigéré A, Naïssengar S, Ndoutamia A G, Zinsstag J, (2003a). Incidence of canine rabies in Ndjamena, Chad. Preventive Veterinary Medicine, (61): 227-233.

[6] Kayali U, Mindekem R, Yemadji N, Kanika Y, Ndoutamia AG Zinsstag J, (2003b). Coverage of pilot parenteral vaccination campaign against canine rabies in Ndjamena, Chad. Bulletin of the World Health Organization, (81): 739-744.

[7] Dürr S, Mindekem R, Kanika Y, Doumagoum Moto D, Meltzer MI, Vounatsou P, et al, (2009). Effectiveness of dog rabies vaccination programmes: comparison of owner-charged and free vaccination campaigns. Epidemiology and Infection, 137(11): 1558-67.

[8] Bourhy H, Rollin PE, Vincent J, Sureau P, (1989). Comparative field evaluation of the fluorescent antibody test, virus isolation from tissue culture, and enzyme immunodiagnosis for rapid laboratory diagnosis of rabies. J Clin Microbiol, 27: 519-23.

[9] Bidjeh K, Assandi O, Saboun M, Lechenne M, Kemdongarti N S, Mindekem R et al. (2014). Resultats de la champagne de vaccination massive des chiens et autres animaux de compagnie contre la rage dans la ville de N"Djamena: 2012. Bulletin of Animal Health and Production in Africa, 62(2), 121-127.

[10] Coleman P G and Dye C (1996). 70\% vaccination coverage is sufficient for rabies elimination. 
[11] Pascu Atanasiu et al. Rabies: Laboratory Techniques (WHO), 2 nd Edition, 1967, 183 pp.

[12] laboratoire de recherches veterinaires et zootechniques de farcha-LRVZ (1992). Annual report, 7-10.

[13] Kayali U et al. (2003). Surveillance of parenteral vaccination campaign against canine rabies, bulletin of WHO, 81, 10: 739744 .

[14] Kayali U, Mindekem R, Yemadji N, Vounatsou P, Kaninga Y, Ndoutamia AG, Zinsstag J, (2003b). Coverage of Pilot Parenteral Vaccination Campaign against Canine Rabies in N'Djamena, Chad. Bulletin of the World Health Organization, 81, 739-744.

[15] Kayali U, Mindekem R, Hutton G, Ndoutamia AG, Zinsstag J. (2006). Cost-Description of a Pilot Parenteral Vaccination Campaign against Rabies in Dogs in N'Djamena, Chad. Too much. Med. Int. Health, 11, 1058-1065.

[16] Mindekem R, U Kayali, Yemadji N, Ndoutamia A G, Zinsstag $\mathrm{J}$, (2005). Impact of canine demography on rabies transmission in N'djamena, Chad. Med Trop, 65 (1): 53-8.

[17] Mindekem R, Lechenne S M, Assandi Oussiguere, Kemdongarti Naissengar S, Bidjeh Kebkiba et al (2017). PostExposure-Prophylaxis and canine mass vaccination against rabies in N'Djamena, Chad. Forehead. Vet. Sci. 4: 38.

[18] Dirr S, Naisssar S K, Mindekem R, Diguimbye C, Niezgoda M, Kuzmin I, et al. Rabies diagnosis for developing countries. PLoS Negl Trop Dis. 2008; 2 (3): E206.

[19] Durr S, Mindekem R, Kanika Y, Doumagoum Moto D, Meltzer MI, Vounatsou P, et al. (2009). Effectiveness of dog rabies vaccination programs: comparison of owner-charged and free vaccination campaigns. Epidemiology and Infect. Nov. 137 (11): 1558-67.

[20] Hampson K, Coudeville L, Lembo T, Sambo M, Kieffer A, Atlan $\mathrm{M}$, et al. Estimating the global burden of endemic canine rabies. PLoS Negl Trop Dis (2015) 9 (4): e0003709.

[21] Hampson K, Dushoff J, Cleaveland S, Haydon DT, Kaare M,
Packer C, et al., (2009). Transmission dynamics and prospects for the elimination of canine rabies. PLoS Biol. 10, 7 (3): 53.

[22] Jackson A C. (2013). Current and future approaches to the therapy of human rabies. Antiviral Res. 99 (1): 61-7).

[23] Knobel D L, Cleaveland S, Coleman P G, Smith E M, Meltzer M I, M E. G. Miranda, et al., (2005). Re-evaluating the burden of rabies in Africa and Asia. Bull. World Health Organ, 83 (5): 360-368.

[24] Cleaveland S, Kaare M, Tiringa P, Mengeya T, Barrat J, (2003). A rabies vaccination campaign in rural Africa. Impact on the incidence of dog rabies and human dog-bite injuries. Vaccine, (21): 19 65-1973.

[25] Frey J, Mindekem R, Kessely H, Doumagoum M D, Naissengar K S, Zingstag J, Schelling E (2013). Survey of animal bite injuries and their management for an estimate of human rabies deaths in N'Djamena. Tropical Medecine and international health n/a-n/a.

[26] Lechenne M S, Naissengar S K, Lepelletier et al. (2016a). Validation of a rapid rabies diagnostic tool for field surveillance in Developping countries. PLoS Negl Tropical diseases 10, e0005010.

[27] Lechenne M S, Oussiguere A, Naissengar K S, Mindekem R, Mosimann L, Rives G et al (2016b). Operationnal performance and analysis of two rabies vaccination campaigns in N'Djamena. Vaccine 34, 571-577.

[28] Sirol J and Provost A (1971). Theoritical data for practical knowledge: 6 cases of human rabies in Fort Lamy. Medecine tropical $31(5), 525-533$.

[29] The urban veterinary clinic of N"Djamena (1990-1994). Annual reports.

[30] Cleaveland S., Fèvre E M, Magai K, Coleman P G (2002). Estimating human rabies mortality in the United of Republic o Tanzania from dog bite injuries. Bull. World Health Organization, 80 (4): 304-310. 